\title{
Determinación de la velocidad óptima para la soldadura MIG de la aleación 5086-H116, mediante ensayo de dureza, prueba de tracción y examen metalográfico para la fabricación de tanqueros de aluminio
}

\section{Determination of the optimum speed for MIG welding of alloy 5086-H116, by hardness test, tensile test and metallographic examination for the manufacture of aluminum tankers}

\author{
José Cabrera Escobar ${ }^{1}$, Raúl Cabrera Escobar ${ }^{1}$
}

\begin{abstract}
Resumen:
Para la construcción de las probetas soldadas por el proceso GMAW de la aleación de aluminio 5086-H116 se utilizaron velocidades de avance $300,400,500,600$ y $700 \mathrm{~mm} / \mathrm{min}$ que están dentro del rango recomendado, las mismas que permitieron variar la energía aportada a la unión. De los resultados obtenidos en los diferentes ensayos se determinó que la velocidad de avance de la soldadura más adecuada es $500 \mathrm{~mm} / \mathrm{min}$ (con una energía aportada de $335,34 \mathrm{~J} / \mathrm{mm}$ ); esta energía es la que permitió obtener las mejores propiedades mecánicas en la soldadura de la aleación 5086- $\mathrm{H} 116$ a un amperaje de 148 A. Con este nivel de energía, se produce la menor pérdida de magnesio, el tamaño de las partículas de $\mathrm{Al}_{3} \mathrm{Mg}_{2}$ es más fino y se distribuyen de manera uniforme en la matriz $\alpha$, lo cual permite obtener las mejores propiedades mecánicas.
\end{abstract}

Palabras clave: avance; energía; evaporación; GMAW.

\begin{abstract}
:
For the construction of the specimens welded by the GMAW process of the aluminum alloy 5086-H116, advance speeds of $300,400,500,600$ and $700 \mathrm{~mm} / \mathrm{min}$ are used that are within the recommended range, the same as they allowed the energy contributed to the union. From the results obtained in the different tests, it was determined that the most suitable welding advance speed is $500 \mathrm{~mm} / \mathrm{min}$ (with a contributed energy of $335.34 \mathrm{~J} / \mathrm{mm}$ ), this energy is the most suitable mechanical in the 5086- $\mathrm{H} 116$ alloy welding at an amperage of $148 \mathrm{~A}$. At this energy level, the lowest loss of magnesium occurs; the particle size of $\mathrm{Al}_{3} \mathrm{Mg}_{2}$ is finer and easier distributed evenly on the a matrix, that allows obtaining the best mechanical properties.
\end{abstract}

Keywords: forward; energy; evaporation; GMAW.

\section{Introducción}

A nivel mundial, la mayoría de empresas dedicadas a la construcción de tanques y equipos de transporte a granel han realizado una serie de investigaciones con el fin de mejorar la calidad constructiva de los mismos y han llegado a la conclusión que una de las alternativas para lograr este objetivo sería cambiar el material que se ha utilizado para la fabricación de este tipo de equipos; reemplazar el acero por el aluminio, por sus múltiples ventajas como su baja densidad. El aluminio es conocido también por su resistencia a la corrosión frente al aire, al agua, los aceites, los alimentos y muchos agentes químicos (Alustock, 2013). Esta resistencia se debe a la existencia de una capa de óxido de aluminio, llamada alúmina, que impide la corrosión del metal (Askeland,

\footnotetext{
${ }^{1}$ Escuela Superior Politécnica de Chimborazo, Riobamba, Ecuador ( \{jose.cabrera, raul.cabrerae\} @espoch.edu.ec).
} 
2012). Se trata de una capa refractaria, que posee una temperatura de fusión muy elevada por lo cual se debe retirar antes o durante la operación de soldeo para permitir una buena fusión del metal base (Solvay, 2012).

En Europa, las aleaciones de aluminio son ampliamente utilizadas para la fabricación de tanqueros de transporte de combustible; para esta aplicación se recomiendan las aleaciones: 5059, 5083, 5086, 5088, 5182, 5186,5383, 5454, 5754 (European, 2011). Los aluminios de la serie 5xxx, son aleaciones de aluminio magnesio endurecidas por dos mecanismos, solución sólida y deformación (Unfried, 2017).

En Ecuador se ha realizado un estudio para determinar el amperaje óptimo de la aleación sometida al proceso y se llegó a la conclusión que el mismo es de 148 A. Debido a que ya se tiene un estudio previo con la aleación 5086- $\mathrm{H} 116$ en el cual se determina su amperaje óptimo, se la escoge para determinar la velocidad optima de soldadura y complementar con esto el estudio previo.

Este estudio ha considerado el sistema de soldadura de arco metálico protegido por gas (GMAW) que utiliza un electrodo consumible y un gas inerte (Rowe, 2008). Como consecuencia de su utilización se obtienen resultados de mejor calidad en las características de la soldadura, que puede ser utilizada para las industrias automotriz, criogénica, marina, tanques de almacenamiento y recipientes de presión; esto podría permitir la utilización más amplia de esta aleación, lo cual sería sin lugar a dudas un aporte a la calidad de los procesos productivos e industriales en Ecuador. En esta investigación se pretende encontrar la velocidad de avance de la soldadura de la aleación 5086-H116, con el objetivo de tener las mejores propiedades mecánicas; para esto se utilizan: ensayo de dureza, prueba de tracción y examen metalográfico; además se podrá obtener un procedimiento que permita conseguir los parámetros óptimos en procesos de soldadura MIG.

\section{Metodología}

Para llevar adelante esta investigación se utilizó el método de inducción científica; se estudiaron los parámetros que influyen en el proceso de soldadura y sus relaciones, así como también se realizaron análisis comparativos para determinar la mejor velocidad de avance.

Para iniciar el proceso de soldadura es necesario contar con el equipo de soldadura MIG, en donde regulamos los parámetros al tomar en consideración el amperaje óptimo de 148 A (Hidalgo, 2013).

Las probetas fueron construidas con planchas de $4,78 \mathrm{~mm}$ de la aleación de aluminio-magnesio $5086-\mathrm{H} 116$ cortadas con plasma para obtener sus medidas recomendadas. En el proceso de soldadura se utilizó un pirotomo, cuyas características se muestran en la Tabla 1, para ejecutar la operación y garantizar la velocidad de avance constante.

Tabla 1. Características del pirotomo

\begin{tabular}{l|l}
\hline Marca & Koike \\
Serie & IK-12 Beetle \\
Voltaje & AC 12 V
\end{tabular}

Se utilizaron electrodos ER5356 que tienen aluminio, con un 5\% de magnesio y un 0,3 de manganeso más cromo; este electrodo es recomendado para aleaciones que tengan de 3 a $5 \%$ de magnesio; al tener la aleación $5086-\mathrm{H} 116$ de 4,5 a $5,5 \%$ de magnesio se elige este electrodo (Soto, 2013).

La calificación del procedimiento de soldadura se realizó de acuerdo con lo especificado en la norma AWS D1.2:2003 (Amú, 2009). 
Para poder cuantificar la energía aportada a la soldadura, se utiliza la Ecuación 1.

$$
\mathrm{HI}=\frac{\beta \times U \times A}{\mathrm{Vs}}
$$

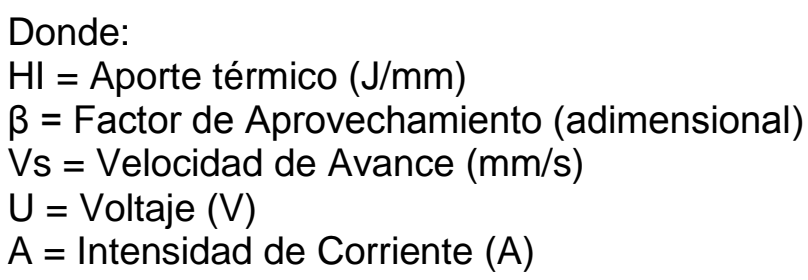

El ensayo de dureza se realizó en el micrómetro Vickers; es importante este ensayo debido a que hay una correlación entre esta propiedad y la resistencia a la tensión (Marulanda, 2013). Se tomaron mediciones de dureza en la ZAC, dado que en esta zona existen variaciones de esta propiedad. Para la medición de dureza se utilizó la norma ASTM de microdureza Vickers (ASTM Committee, 2017), el micrómetro debe ser calibrado antes de su utilización para garantizar sus mediciones (Espín, 2017).

La prueba de tracción se realizó en la máquina universal, controlando los desplazamientos de cada probeta (Naranjo, 2013). Los ensayos de tracción se efectuaron siguiendo el procedimiento establecido en la norma ASTM para aleaciones de aluminio magnesio (ASTM Committee, 2015). Se realizaron tres probetas por cada velocidad de avance (Piccini, 2017); a partir de los resultados de estas tres probetas, se realizó un promedio para encontrar las propiedades de: porcentaje de elongación, porcentaje de reducción de área, módulo de elasticidad, resistencia a la cedencia y resistencia a la tracción (Navas, 2017).

Para el examen metalográfico se utilizó el microscopio óptico para tomar las microfotográfias en el centro de la ZAC; las probetas fueron pulidas en calidad de espejo y atacadas con Keller durante 20 segundos (Cabrera, 2014). Para realizar la microscopia óptica se siguió el procedimiento establecido en la norma ASTM para preparación de probetas sometidas a la mencionada prueba (ASTM Committee, 2017).

Se realizaron estas tres pruebas debido a que la resistencia, la dureza y la microestructura en la ZAC son diferentes a las que presenta el material base (Fuentes, 2016).

\section{Resultados}

Las condiciones utilizadas en el proceso de soldadura de la aleación son las que se muestran en la Tabla 2.

Tabla 2. Registro de los ensayos de soldadura

\begin{tabular}{|c|c|c|c|}
\hline \multicolumn{4}{|c|}{ Formato de registro de ensayo de soldadura } \\
\hline Tipo de gas & Argón & Presión de gas & $5 \mathrm{psi}$ \\
\hline Amperaje & $148 \mathrm{~A}$ & Voltaje & $25 \mathrm{~V}$ \\
\hline Velocidad de Avance & $550 \mathrm{~mm} / \mathrm{min}$ & Diámetro del alambre & $1,2 \mathrm{~mm}$ \\
\hline \multicolumn{4}{|c|}{ Composición química del alambre } \\
\hline $\mathrm{Mn}$ & $\mathrm{Si}$ & $\mathrm{Cr}$ & $\mathrm{Ti}$ \\
\hline $0,05-0,20 \%$ & $0,25 \%$ & $0,05-0,20 \%$ & $0,06-0,20 \%$ \\
\hline $\mathrm{Mg}$ & $\mathrm{Zn}$ & $\mathrm{Fe}$ & \\
\hline $4,5-5,5 \%$ & $0,10 \%$ & $0,40 \%$ & \\
\hline
\end{tabular}




\section{Cálculo de la energía aportada a la soldadura}

En la Tabla 3 se muestran los resultados de la energía aportada a la soldadura. Se deduce que a menor velocidad de avance mayor el aporte de energía y mientras mayor sea la velocidad de avance menor el aporte. El cálculo de la energía es importante debido a que los cambios microestructurales se deben a la energía que se aporta al soldar (Sánchez-2016).

Tabla 3. Energía aportada a la soldadura en las diferentes velocidades de avance

\begin{tabular}{c|c}
$\begin{array}{c}\text { Velocidad de Avance } \\
(\mathrm{mm} / \mathrm{min})\end{array}$ & $\begin{array}{c}\text { Energía Aportada a la } \\
\text { Soldadura }(\mathrm{J} / \mathrm{mm})\end{array}$ \\
\hline 300 & 592,00 \\
400 & 443,78 \\
500 & 355,34 \\
600 & 296,00 \\
700 & 253,64
\end{tabular}

\section{Ensayo de tracción}

La Figura 1 corresponde a la curva tensión-deformación que se obtuvo en la máquina universal; con esta curva se lograron las características de la probeta que se presenta en la Tabla 4. En la curva esfuerzo-deformación se puede observar la gran ductilidad que tiene la soldadura de la aleación, debido a que presenta un 8,36\% de deformación antes de la fractura; su esfuerzo de fractura es de $24,1 \mathrm{Kg} / \mathrm{mm}^{2}$.

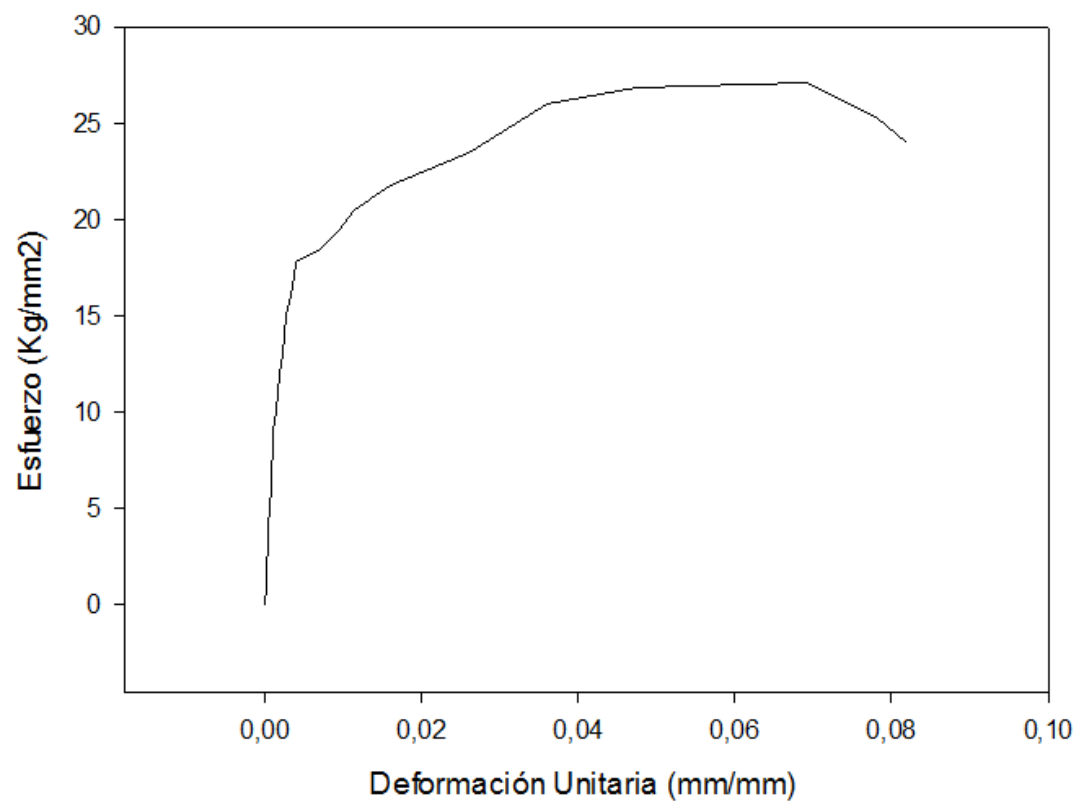

Figura 1. Curva tensión-deformación probeta 500-1

Tabla 4. Características obtenidas de la curva tensión-deformación de la probeta 500-1

\begin{tabular}{c|c|c|c|c}
$\begin{array}{c}\text { Porcentaje de } \\
\text { elongación }\end{array}$ & $\begin{array}{c}\text { Porcentaje de } \\
\text { reducción de } \\
\text { área }\end{array}$ & $\begin{array}{c}\text { Módulo de } \\
\text { elasticidad } \\
\left(\mathrm{kg} / \mathrm{mm}^{2}\right)\end{array}$ & $\begin{array}{c}\text { Resistencia a la } \\
\text { cedencia Sy } \\
\left(\mathrm{kg} / \mathrm{mm}^{2}\right)\end{array}$ & $\begin{array}{c}\text { Resistencia a la } \\
\text { tracción Sut } \\
\left(\mathrm{kg} / \mathrm{mm}^{2}\right)\end{array}$ \\
\hline 8.07 & 6.05 & 7873.00 & 17.37 & 27.18
\end{tabular}

De la superficie de fractura de la probeta 500-1 de la Figura 2, se puede determinar que el tipo de fractura es dúctil porque la fractura alcanza un ángulo de $45^{\circ}$ respecto al 
eje normal de tracción; no toda el área de la fractura está a $45^{\circ}$ pero sí se puede observar que en su gran mayoría.

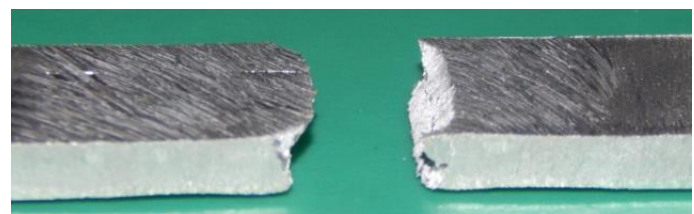

Figura 2. Aspecto de la fractura de la probeta 500-1

\section{Prueba de dureza}

En la Figura 3 se puede observar, de la misma forma que en todas las probetas, que en el centro del cordón la dureza es menor; en este caso la dureza es de 89,1 HV y poco a poco se incrementa a medida que la distancia aumenta respecto al eje de soldadura; alcanza los $98 \mathrm{HV}$ a una distancia de $33 \mathrm{~mm}$ y luego se estabiliza. Esto indica que la zona afectada por el calor (ZAC) alcanza los $33 \mathrm{~mm}$.

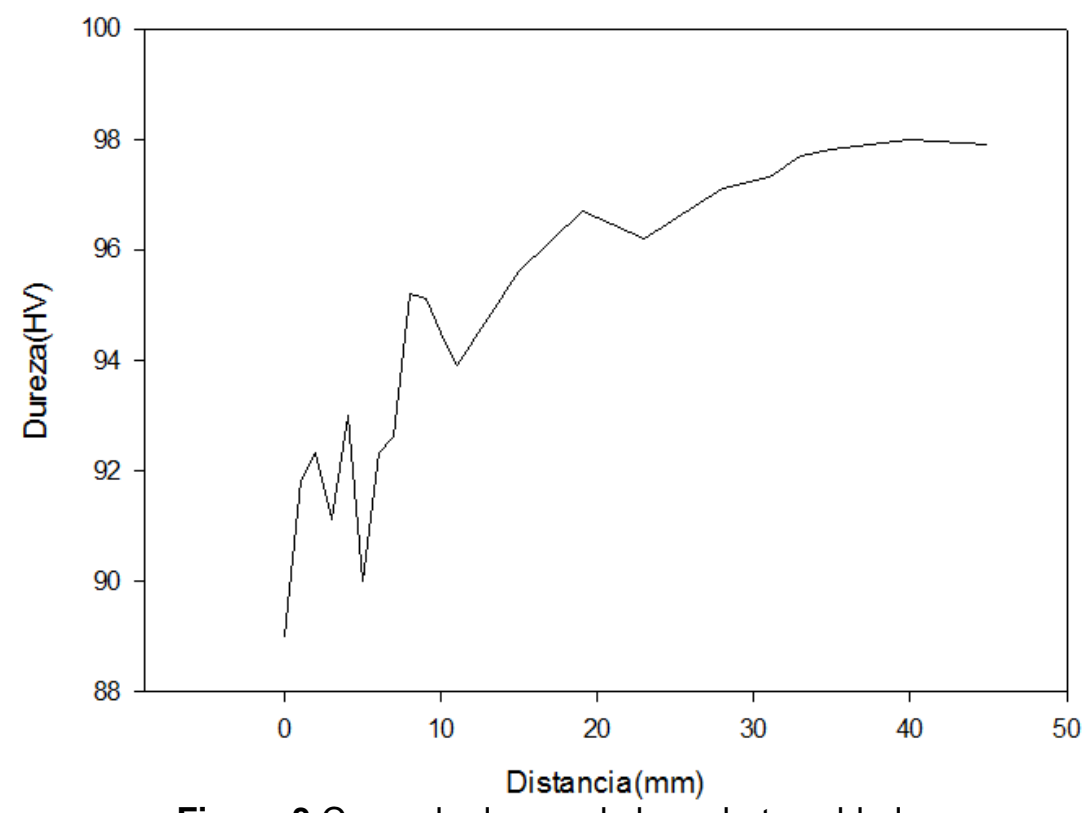

Figura 3.Curva de dureza de la probeta soldada.

\section{Examen metalográfico}

En la microfotografía de la Figura 4 se puede observar que la matriz corresponde a la solución sólida a y las pequeñas partículas grises al constituyente $\mathrm{Al}_{3} \mathrm{Mg}_{2}$, en menor cantidad que en el metal base debido a la pérdida de magnesio durante la fusión, pero su distribución es uniforme, por lo cual las propiedades de la soldadura no se ven seriamente afectadas como en los otros casos; en esta aleación también se tienen impurezas como el silicio y el hierro (Planella, 2005). 


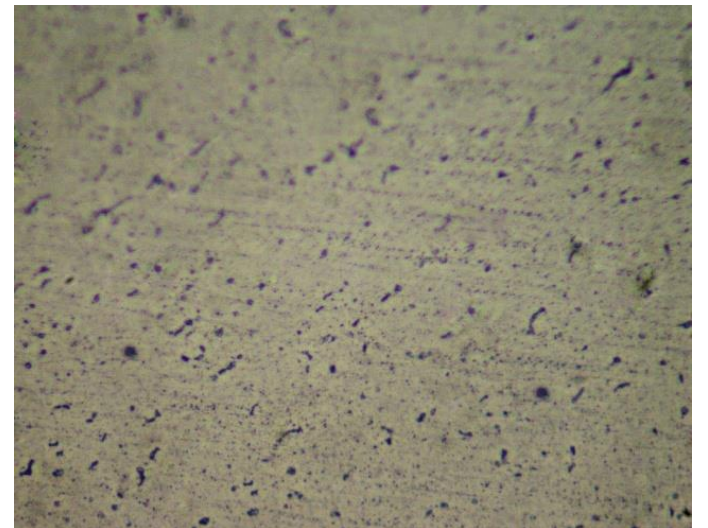

Figura 4. Microfotografía de material soldado a una velocidad de avance de $500 \mathrm{~mm} / \mathrm{min}$.

Se determinó que a $500 \mathrm{~mm} / \mathrm{min}$ el porcentaje del $\mathrm{Al}_{3} \mathrm{Mg}_{2}$ es de $2,6 \%$ y el porcentaje de la matriz a es de $97,4 \%$.

En la Figura 5 se puede observar que las partículas grises, correspondientes al $\mathrm{Al}_{3} \mathrm{Mg}_{2}$, son más grandes y no se distribuyen uniformemente en la matriz $\alpha$.

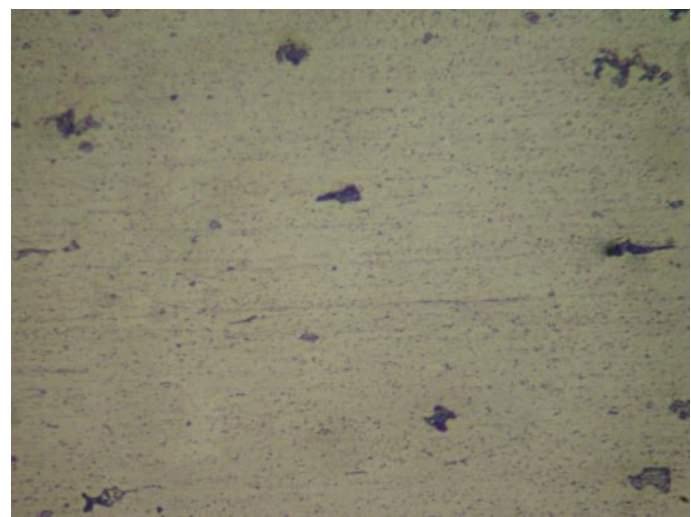

Figura 5. Microfotografía de material soldado a una velocidad de avance de $300 \mathrm{~mm} / \mathrm{min}$.

A continuación, la Tabla 5 presenta un resumen de las diferentes pruebas realizadas a las probetas; se puede observar que la probeta con las mejores propiedades tiene una velocidad de avance de $500 \mathrm{~mm} / \mathrm{min}$.

Tabla 5. Resumen comparativo de propiedades mecánicas obtenidas a diferentes velocidades de avance

\begin{tabular}{c|c|c|c|c|c|c}
$\begin{array}{c}\text { Velocidades } \\
\text { de avance } \\
(\mathrm{mm} / \mathrm{min})\end{array}$ & $\begin{array}{c}\text { Resistencia } \\
\text { a la } \\
\text { tracción } \\
\left(\mathrm{Kg} / \mathrm{mm}^{2}\right)\end{array}$ & $\begin{array}{c}\text { Porcentaje de } \\
\text { reducción de la } \\
\text { resistencia a la } \\
\text { tracción } \\
\text { respecto a la } \\
\text { velocidad de } \\
\text { avance de } 500 \\
\mathrm{~mm} / \mathrm{min}\end{array}$ & $\begin{array}{c}\text { Resistencia a } \\
\text { la cedencia } \\
\left(\mathrm{Kg} / \mathrm{mm}^{2}\right)\end{array}$ & $\begin{array}{c}\text { Porcentaje de } \\
\text { reducción de la } \\
\text { resistencia a la } \\
\text { cedencia } \\
\text { respecto a la } \\
\text { velocidad de } \\
\text { avance de } 500 \\
\text { mm/min }\end{array}$ & $\begin{array}{c}\text { Reducción } \\
\text { área }(\%)\end{array}$ & $\begin{array}{c}\text { Elongación } \\
(\%)\end{array}$ \\
\hline 300 & 25,67 & 5,10 & 16,39 & 8,07 & 4,61 & 6,52 \\
400 & 26,08 & 3,59 & 16,96 & 4,88 & 3,99 & 5,93 \\
500 & 27,05 & - & 17,83 & - & 6,79 & 8,36 \\
600 & 24,65 & 8,87 & 16,68 & 6,45 & 6,56 & 4,85 \\
700 & 24,75 & 8,50 & 15,99 & 10,32 & 4,74 & 4,99
\end{tabular}




\section{Discusión}

De los resultados obtenidos en los diferentes ensayos, se determinó que la velocidad de avance óptima es de $500 \mathrm{~mm} / \mathrm{min}$ (con un energía aportada de 355,34 $\mathrm{J} / \mathrm{mm}$ ); a esta velocidad se obtienen las mejores propiedades mecánicas, además en esta velocidad de avance se produce la menor pérdida de magnesio, el tamaño de las partículas de $\mathrm{Al}_{3} \mathrm{Mg}_{2}$ es más fino y se distribuyen de manera uniforme en la matriz $\alpha$.

A velocidades menores, esto es 300 y $400 \mathrm{~mm} / \mathrm{min}$, la energía aportada es demasiado elevada lo cual produce una gran pérdida de $\mathrm{Mg}$ por evaporación, tanto en la solución sólida $\alpha$, como en la fase intermetálica $\mathrm{Al}_{3} \mathrm{Mg}_{2}$. A velocidades mayores, a 550 , 600 y $700 \mathrm{~mm} / \mathrm{min}$, la energía aportada es insuficiente para disolver las partículas de $\mathrm{Al}_{3} \mathrm{Mg}_{2}$ y conseguir una distribución uniforme en la matriz $\alpha$.

Es importante resaltar la influencia positiva sobre las propiedades mecánicas que tienen la cantidad, el tamaño y la distribución de las partículas intermetálicas de $\mathrm{Al}_{3} \mathrm{Mg}_{2}$. Por lo tanto, el proceso de soldadura se debe realizar bajo parámetros que permitan que las partículas de segunda fase se hallen en condiciones que brinden las mejores propiedades en la unión.

\section{Conclusiones y recomendaciones}

Se estudió el efecto de la energía en la soldadura GMAW de la aleación 5086-H116 sobre la microestructura y propiedades mecánicas de resistencia y dureza. El nivel de energía que permitió obtener los mejores resultados es $355,34 \mathrm{~J} / \mathrm{mm}$, el cual se obtuvo con una velocidad de avance de la soldadura de $500 \mathrm{~mm} / \mathrm{min}$.

Se determinaron los cambios microestructurales en las juntas soldadas y su efecto en las propiedades mecánicas de resistencia y dureza, concluyendo que son importantes el porcentaje, el tamaño y la distribución de la fase intermetálica $\mathrm{Al}_{3} \mathrm{Mg}_{2}$.

Con los resultados provenientes de esta investigación se pueden poner a disposición de la industria los parámetros óptimos de soldadura para la aleación 5086$\mathrm{H} 116$ con proceso GMAW, que son un amperaje de $148 \mathrm{~A}$, voltaje de $25 \mathrm{~V}$ y una velocidad de avance de $500 \mathrm{~mm} / \mathrm{min}$.

\section{Bibliografía}

Alustock. (2013). El libro del Aluminio. $5^{\text {ta} E d . ~ E d i t o r i a l ~ A l u s t o c k . ~ M a d r i d, ~ E s p a n ̃ a . ~}$

Amú, M., F. Franco. (2009). Microestructura y propiedades mecánicas en la zona afectada por el calor de la unión soldada de la aleación 6261-T5. Suplemento de la Revista Latinoamericana de Metalurgia y Materiales. S1(2):767-772.

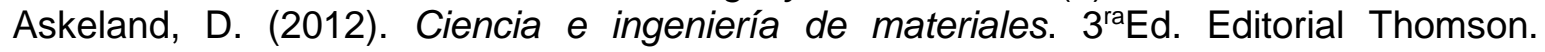
Toronto, Canadá.

ASTM Commitee. (2015). ASTM B557. Standard Test Method for Tension Testing Wrought and Cast Aluminum-and-Magnesium-Alloy Products. $3^{\text {ra }} E d$. ASTM Commitee. West Conshohocken, USA.

ASTM Commitee. (2017). ASTM E3. Standard Practice for Preparation of Metallographic.

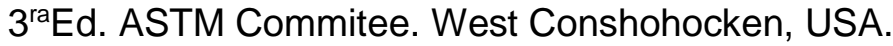

ASTM Commitee. (2017). ASTM E92. Standard Test Methods for Vickers Hardness of

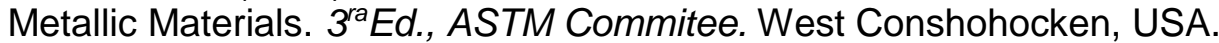

Cabrera, J. O., y M. Almendáríz. (2014). Influencia de la energía aportada a la soldadura GMAW de la aleación 5086-H116 sobre la microestructura y propiedades mecánicas de resistencia y dureza. Tesis de Grado de Ing. Mecánica. Escuela Superior Politécnica de Chimborazo. Riobamba, Ecuador.

Espín, S., J. Guamanquispe, D. Coello, J. Paredes. (2017). Blindaje por proyección térmica en flancos de dientes de un virador de molino de caña de ingenio azucarero. Enfoque UTE. ISSN: 1390-6542. 
European Aluminium Association. (2011). Aluminium in Commercial Vehicles, $1^{\text {ra }}$ Ed. Editorial European Aluminium Association. Bruselas, Bélgica.

Fuentes, R., T. Fernández, O. Gámez, N. Durán. (2016). Microestructura de una unión disímil AISI 321 y una aleación A351 grado HK-40 obtenida mediante soldadura manual por arco eléctrico. Revista Minería y Geología. ISSN. 19938012.

Hidalgo, F., y R. Cabrera. (2013). Estudio de la Variación del Amperaje al realizar soldaduras a tope mediante proceso GMAW aplicado a la aleación de aluminio 5086-H116 y su efecto en las propiedades mecánicas de la zona afectada por el calor. Tesis de Maestría de Diseño Mecánico. Universidad Técnica de Ambato. Ambato, Ecuador.

Marulanda, J., V. Burbano, J. Peláez. (2013). Análisis de la Soldabilidad de aceros inoxidables con aceros de medio y bajo carbono por SMAW. Revista Facultad de Ingeniería. UPTC. ISSN 0121-1129.

Naranjo, A., R. Rodríguez, J. Arroyo. (2013). Caracterización de la transformación inducida por deformación plástica en aceros $0,23 \% \mathrm{C}-1,11 \% \mathrm{Mn}-0,23 \% \mathrm{Ni}-0,68 \% \mathrm{Cr}$. Revista ITECKNE. ISSN 1692-1798.

Navas, H., W. Vaca, J. Paredes, F. Morales, D. Núñez. (2017). Análisis cuantitativo de los elementos aleantes principales precipitados en billets de aluminio de aleación 6063 en equipo de fundición de colada continua horizontal para el proceso de extrusión. Revista de Ciencia y Tecnología INGENIUS. ISSN: 1390-860X

Piccini, J., H. Svoboda. (2017). Efecto de la velocidad de rotación y la indentación en soldadura de punto por fricción agitación de aleaciones de aluminio disimilares. Revista de Metalurgía. ISSN-L:0034-8570.

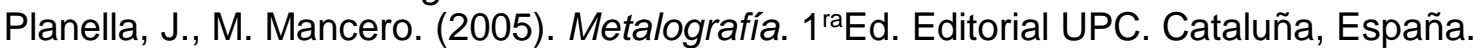

Rowe, R. (2008). Manual de Soldadura Gmaw(Mig/Mag). Madrid, España.

Sánchez, W.B. (2016). Efecto térmico de la soldadura por arco sumergido en un acero bajo aleado. Revista Ciencia \& Futuro. ISSN. 2306-823X.

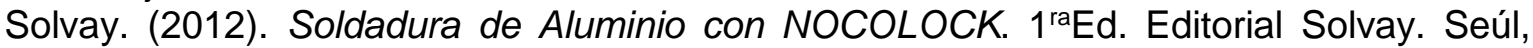
Corea del Sur.

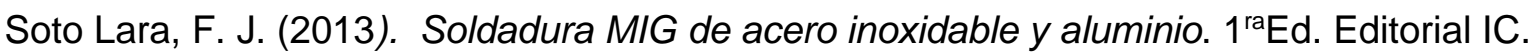
Málaga, España.

Unfried, J., J. López, D. Machuca, E. Caicedo, C. Zambrano, E. Niebles. (2017). Efectos de la aplicación de tratamientos isotérmicos sobre el tamaño de grano y la dureza de aleaciones de aluminio AA5083-H116. Revista Materia. ISSN 1517-7076. 